\title{
DIGITAL NATIVES AND DIGITAL IMMIGRANTS: DIFFERENCES IN ONLINE TRUST FORMATION
}

\author{
CHRISTIAN PIETER HOFFMANN \\ University of St. Gallen \\ 9000 St. Gallen \\ Switzerland \\ ANDREA VON KAENEL \\ University of St. Gallen \\ CHRISTOPH LUTZ \\ University of St. Gallen \\ MIRIAM MECKEL \\ University of St. Gallen
}

\section{INTRODUCTION}

Online trust research has come a long way in analyzing the multi-faceted trust construct and identifying antecedents and drivers of online trust (Culnan \& Armstrong, 1999; Milne \& Boza, 1999; Hoffman et al., 1999a; Milne, 2000; Jarvenpaa et al., 2000; Bart et al., 2005). A range of authors have proposed that user characteristics play an important role in online trust formation. Web experience has been argued to affect various elements of online trust and users' willingness to engage in specific transactions (Urban et al., 2009; McKnight et al., 2002a; Shankar, Urban, \& Sultan, 2002; Hoffman et al., 1999a; Walczuch \& Lundgren, 2004). Other authors have focused on demographic user characteristics, theorizing that factors such as age, sex, educational attainment, and employment status might impact trust formation (Phelps, Nowak \& Ferrell, 2000; Lee \& Turban, 2001; Bhatnagar, Misra, \& Rao, 2000; Chen \& Rea, 2004; Sheehan, 1999).

Based on a survey of German internet users, our study picks up on these propositions and analyzes variances in online trust formation between groups with different user characteristics. To this end, we propose a model of online trust formation, which encompasses key trust antecedents as well as so-called trust drivers (McKnight et al., 2000; 2002a; Gefen, 2000; Bhattacherjee, 2002; Jarvenpaa et al., 2000; Bart et al., 2005). We conducted a latent class analysis based on web experience and demographic user characteristics in order to differentiate groups with specific features, resulting in two distinct user groups. A subsequent multiple group analysis of the proposed model then served to identify variances in online trust formation between both groups.

\section{RESEARCH MODEL}

\section{Online Trust}

Rousseau, Sitkin, Burt and Camerer (1998, p. 395) proposed a widely accepted definition of the trust concept as "a psychological state comprising the intention to accept vulnerability based upon positive expectations of the intentions or behavior of another". When judging the trustworthiness of an interaction partner online, individuals particularly take the information or 
cues provided by its web presence into consideration (Gefen, 2000; Hoffman et al., 1999a; Friedman et al., 2000; Jarvenpaa et al., 2000). Bart et al. (2005, p. 134) point out: "online trust includes consumer perceptions of how the site would deliver on expectations, how believable the site's information is, and how much confidence the site commands."

Figure 1 about here

McKnight et al. (2002a) proposed a helpful elaboration of the online trust construct by differentiating "trusting beliefs", i.e. perceptions of a trustee's attributes that are deemed beneficial to the truster, from "trusting intentions", i.e. the truster's willingness to depend on the trustee. Trusting beliefs are conceptualized to promote trusting intentions. Also, the authors (p. 339) identified "institution-based trust", understood as "the belief that needed structural conditions are present (e.g., in the Internet) to enhance the probability of achieving a successful outcome", and an individual's "disposition to trust", ", i.e. "a tendency to depend on others across a broad spectrum of situations and persons" as drivers of trusting beliefs and intentions.

A range of website or service features provide users with cues as to the trustworthiness of the transaction partner (Wang et al., 2004; Bart et al., 2005). We conducted a literature review in order to analyze previously identified "trust cues":

Reciprocity: Users are willing to enter a transaction by providing some personal information if the purpose is to gain a clear benefit (Koufaris \& Hampton-Sosa, 2004; Chen \& Barnes, 2007). Fair information or disclosure practices are one prerequisite of reciprocity in a transaction (Culnan \& Armstrong, 1999; Wang et al., 2004; Lanier \& Saini, 2008). Brand \& Reputation: Strong brands are recognized as an important driver of e-business (Corbitt et al., 2003; Ha \& Perks, 2005; Jevons \& Gabbott, 2000; Lowry et al., 2008). Previous studies have found that when it comes to trusting an online service, consumers prefer large and well-established providers (Jarvenpaa et al., 2000; Krishnamurthy, 2001; Luo \& Cook, 2007-2008). User Control: When users fear losing control over the spread and use of their personal data, they lose trust in a transaction partner (Milne \& Boza, 1999; Hoffman et al., 1999b; Phelps, D’Souza, \& Nowak, 2001). Businesses, in turn, can garner trust by providing users control over their personal information (Culnan \& Armstrong, 1999). Offline Presence: The physical appearance of a business - its offices, stores or branches - provides users with a range of cues as to its trustworthiness (Jarvenpaa \& Tractinsky, 1999). Third Party Endorsements: Independent statements of experts and professionals, such as certificates or seals, attest the trustworthiness of a business (Wakefield \& Whitten, 2006; Urban et al., 2000; Cook \& Luo, 2003; Wang et al., 2004). Online businesses also increasingly rely on user ratings, comments, bookmarking or reporting to signal their quality (Pavlou, 2002; Pennington, Wilcox, \& Grover, 2003).

Figure 1 shows the resulting hypothesized model of online trust formation.

\section{User Characteristics}

A range of studies posit that user characteristics influence online trust formation (Urban et al., 2009; Shankar et al., 2002; Bart et al., 2005). McKnight et al. (2002a) propose that general web experience should have a positive impact on trust formation, "because experience provides the consumer with a feeling that dealing with the Web is proper and normal" (p. 340). Corbitt et al. (2003) confirm that more general web experience contributes to increased trust in e-commerce. Hoffman et al. (1999a) show that experienced Internet users more critically consider an online 
retailer's privacy and security standards than less experienced users. In summary, general web experience is held to influence online trust formation.

Aside from web experience, demographic user characteristics have also been proposed as influencers of online trust formation. Phelps et al. (2000) test for an impact of factors such as age, sex, marital status, educational attainment, and employment status on privacy beliefs and concerns in the context of direct marketing, and find that higher levels of education corresponded with reduced privacy concerns. Bhatnagar et al. (2000) analyze the influence of demographics on online shopping-behavior and find that age increases openness to online shopping. Chen and Rea (2004) studied the impact of demographics on the choice of online privacy controls and found differences among genders as well as races.

In order to further explore the impact of user characteristics such as web experience and demographics on online trust formation, our analysis will address the following research question by conducting a multiple group analysis of the research model, based on a latent class analysis:

RQ1: Are there differences in online trust formation based on users' web experience and demographic user characteristics?

\section{METHODOLOGY AND RESULTS}

\section{Sample and Measures}

Our analysis is based on a survey among German Internet users conducted in late 2010. The survey sample was recruited from a pool of Internet users demographically representative of the German population, provided by a leading market research institute. 11'846 users were invited to participate in the survey by e-mail and offered a small monetary incentive. 1'549 users responded (response rate 12.68\%), 379 of whom did not complete the whole questionnaire.

The questionnaire questioned participants on some demographics such as age, gender, education and employment, as well as their Internet usage habits, i.e. length of experience, usage context (private or professional) and frequency (including usage frequency of specific online applications). It also asked participants to judge their own Internet skills based on a few standardized items (Shapiro \& Hughes, 1996; Leung, 2009; Bart et al., 2005; McKnight et al., 2002a). Subjects were then asked to think back to the last time they signed up to a new online service by opening an account which required the provision of some personal data. All questions regarding trusting beliefs, trusting intentions and trust cues then referred to the chosen provider. The scales for the four core trust constructs were derived from McKnight et al. (2000; 2002a), Jarvenpaa et al. (2000), Bart et al. (2005), Urban et al. (2009), Bhattacherjee (2002) and Gefen (2000). The scales for the trust cues were derived from a comprehensive literature review. Each item was rated by the survey participants based on a five-point Likert scale.

\section{Measurement Model}

The measurement model was evaluated based on a confirmatory factor analysis (Anderson $\&$ Gerbing, 1988). On the construct level, we used Cronbach's alpha $(\alpha)$, composite reliability (C.R.) and average variance extracted (AVE) to assess the internal consistency of the scales, all of which were above the required criterion values, except for "offline presence" $(\mathrm{AVE}=0.447)$ and "third party endorsements" (AVE $=0.425$ ). Overall, we can assume scale reliability. Discriminant validity of the constructs can also be assumed, because no correlation between the latent constructs exceeded the squared AVE value. 


\section{Results}

Table 1 presents the structural equation model tested with Mplus for the adjusted sample $(\mathrm{N}=1170)$. The results of this first step include the standardized coefficients based on robust Maximum-Likelihood-estimation (MLM) and the total variance explained $\left(\mathrm{R}^{2}\right)$ for each dependent construct for all participants without missing values. Many of the hypothesized paths were highly significant $(\mathrm{p}<0.01)$ and the whole model provided good fitness indices (Chi-squared/d.f. $=2.72$, $\mathrm{CFI}=0.93, \mathrm{TLI}=0.92, \mathrm{SRMR}=0.057, \mathrm{RMSEA}=0.038)$

In order to identify groups (classes) with similar response patterns concerning demographic and web experience characteristics, we conducted a latent class analysis (LCA). We tested a twoclass-model in comparison to other latent class solutions, i.e. 1 and 3-classes, using Mplus (Muthén \& Muthén, 2006). The analysis provided substantial evidence for a 2-class separation (entropy value of 0.775 ). The p-values of 1.000 for both Pearson Chi-squared and Likelihood Ratio Chisquared indicate that the difference between the observed and implicated response-patterns was insignificant.

A look at the resulting class characteristics reveals significant differences in web or, more specifically, application experience: while all participants use the Internet frequently (daily or several times a week) and have been using it for more than 8 years, they differ in how they use the internet. Members of Class 2 use all applications covered in the survey more frequently than Class 1 (except for online banking). Most significantly, Class 2 is much more active in its use of social networking sites (SNS), blogs and twitter. Online dating and gaming is also more frequent among Class 2. Both classes are most similar in their use of more established or traditional applications like online banking, online shopping, reading online news and e-mailing. When asked for their skills in using the Internet, members of Class 2 are significantly more likely to judge themselves as able to create a profile on a SNS, publish a video online, and information on a blog or micro-blog.

If we look at the demographics of both classes, we do not find any significant gender differences, but age clearly discriminates both classes: $47.9 \%$ of Class 2 are 25 and younger $(86.4 \%$ are under 46 ), $80.7 \%$ of Class 1 are older than 45 . Accordingly, retirees are significantly more likely to belong to Class 1 , while students largely belong to Class 2 . Finally, education also differentiates the two classes: significantly more members of Class 1 named a completed apprenticeship as their highest degree; members of Class 2 are more likely to have finished high school. These distinct class characteristics lead us to name Class 1 "Digital Immigrants" and Class 2 "Digital Natives". Prensky (2001) defined "Digital Natives" as the student generations growing up and immersed in digital technology (cf. Palfrey \& Gasser, 2008). He differentiates this group from "Digital Immigrants", i.e. the Internet user generations that had to adopt digital technologies later in life. Digital Immigrants, while skilled in their use of the Internet, still prefer to conduct many interactions like networking, socializing, or gaming offline.

Finally, to answer our research question, we conducted a multiple-group SEM (MGA) for the two classes identified in the LCA. We tested the model for configural $\left(\mathrm{M}_{1}\right)$, metric $\left(\mathrm{M}_{2}\right)$, and scalar $\left(\mathrm{M}_{3}\right)$ invariance between the two groups and found good fits respectively $\left(\mathrm{M}_{1}\right.$ : Chi-squared/ $\mathrm{df}=2.45, \mathrm{CFI}=0.92, \mathrm{TLI}=0.91, \mathrm{RMSEA}=0.050, \mathrm{SRMR}=0.062 ; \mathrm{M}_{2}:$ Chi-squared $/ \mathrm{df}=2.46$, $\mathrm{CFI}=0.92, \mathrm{TLI}=0.91, \mathrm{RMSEA}=0.050, \mathrm{SRMR}=0.064 ; \mathrm{M}_{3}$ : Chi-squared $\mathrm{df}=2.54, \mathrm{CFI}=0.91$, TLI $=0.91$, RMSEA $=0.051, \mathrm{SRMR}=0.065)$.

Table 1 shows the respective model coefficients for both classes. We can observe some interesting differences in online trust formation: as to the core trust constructs, disposition to trust more strongly influences institution-based trust and trusting beliefs among "Digital Natives". 
Among these more intensive Internet-users, institution-based trust also affects trust more strongly. As to the trust cues, "reciprocity" ( $\left.\beta_{2}=0.367\right)$ and "third-party endorsements" $\left(\beta_{2}=0.381\right)$ play a much more significant role in trust formation among "Digital Natives", while "brand \& reputation" $\left(\beta_{1}=0.406\right)$ and "offline presence" $\left(\beta_{1}=0.208\right)$ impacts trusting beliefs more strongly among "Digital Immigrants".

Table 1 about here

\section{DISCUSSION}

While the construct of Digital Natives vs. Digital Immigrants has proven theoretically contentious, an analysis of our demographically representative sample of German Internet users did yield two distinct user groups: the first group we called "Digital Immigrants", as members of this group were of higher age (largely above 45), exhibited in general lower educational levels, a high proportion of retirees and blue-collar workers. More importantly, though, the web experience of this group, while extensive, is largely limited to traditional online services like shopping, banking and news. In contrast, we named the second user group "Digital Natives", as members of this group are much younger, in many cases students. Educational levels are higher, and white-collar jobs are strongly represented. Social media are an integral part of this second group's web experience.

Our analysis was able to show that when judging the trustworthiness of a service, "Digital Immigrants" strongly rely on well-known and established brands with a large customer base. A simple and professional website design featuring familiar elements and processes also contribute to the trust of this group (cf. Urban et al., 2000; Belanger et al., 2002; Shneiderman, 2000; Gefen \& Straub, 2003). Additionally, the availability of physical outlets strongly contributes to trust formation among "Digital Immigrants". This confirms the notion that Digital Immigrants prefer to conduct a range of their transactions offline, and tend to transfer their offline affiliations to the Web.

"Digital Natives", on the other hand, while also attracted to strong brands and reputable providers, strongly base their trust on the reciprocity of a service. Fair information practices are very important to this group (Culnan \& Armstrong, 1999; Lanier \& Saini, 2008). Trust is built when providers offer a clear benefit that outweighs the risk of a transaction. Businesses targeting this user group should therefore stress the benefits of their offer (Milne \& Boza 1999; Ashworth \& Free, 2006; Olivero \& Lunt, 2004). When targeting "Digital Natives", online businesses should also communicate endorsements such as seals of approval or prizes in order signal their reliability and competence (cf. Urban et al., 2000; Cook \& Luo, 2003; Wang et al., 2004). Rating and social bookmarking sites should also prove a valuable tool when reaching out to social media-savvy "Digital Natives".

Our analysis shows that web experience and demographics, while both individually worth further exploration, in fact interact when differentiating user groups. One advantage of the Digital Native/Immigrant dichotomy therefore lies in the fact that it combines demographic and usage variables. We believe such a combined approach to be a promising addition to online trust research.

\section{REFERENCES AVAILABLE FROM THE AUTHORS}




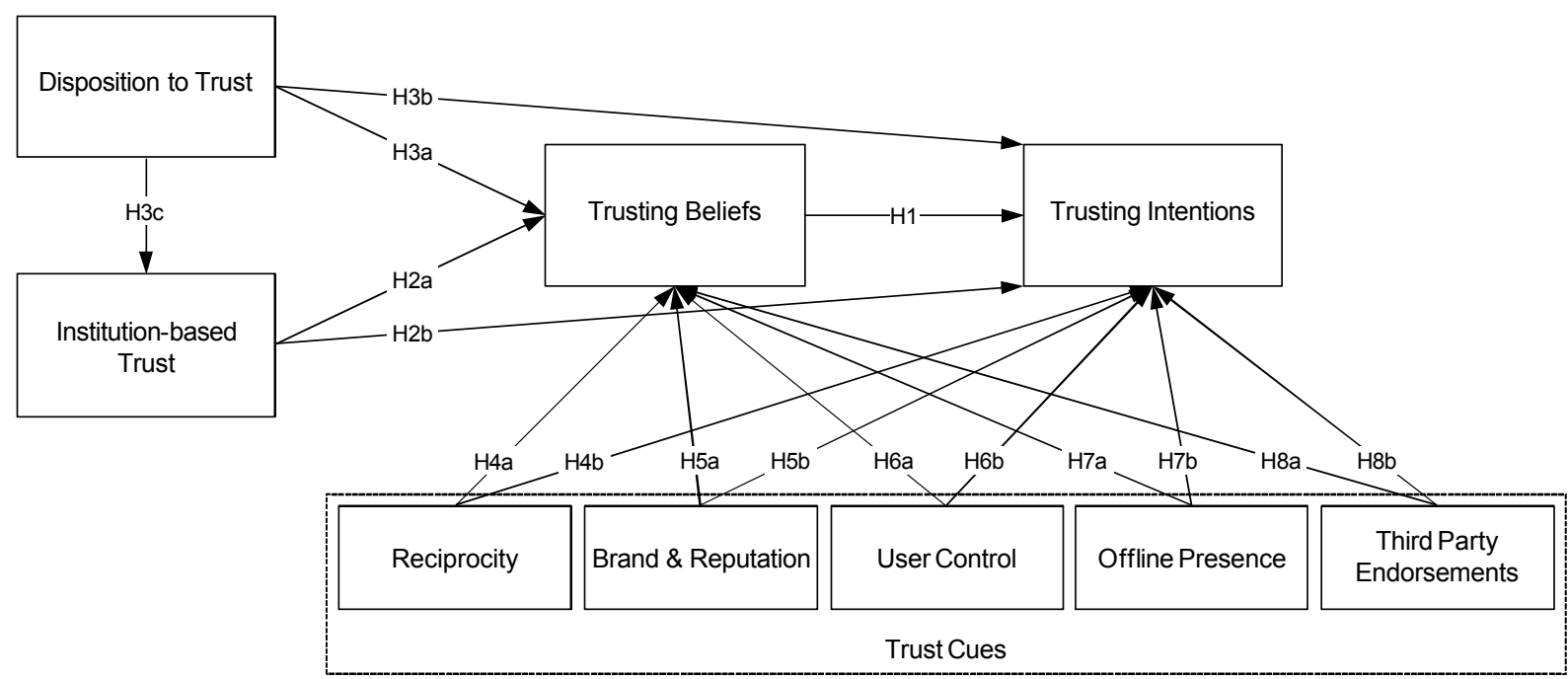

Figure 1: Overview of the Hypotheses in the Model

\begin{tabular}{|l|l|l|l|}
\hline Relationship & $\begin{array}{l}\text { Coefficient Total } \\
\text { Model (t-value) }\end{array}$ & Coefficient Class 1 & Coefficient Class 2 \\
\hline H1: TRUST $\rightarrow$ INT & $0.732(24.908)^{* *}$ & $0.704^{* *}$ & $0.724^{* *}$ \\
\hline H2a: INSTIT $\rightarrow$ TRUST & $0.166(5.878)^{* *}$ & $0.108^{* *}$ & $0.197^{* *}$ \\
\hline H2b: INSTIT $\rightarrow$ INT & $0.014(0.585)$ & 0.050 & -0.041 \\
\hline H3a: DISP $\rightarrow$ TRUST & $0.136(4.412)^{* *}$ & $0.128^{* *}$ & $0.137^{* *}$ \\
\hline H3b: DISP $\rightarrow$ INT & $0.033(1.184)$ & 0.039 & 0.026 \\
\hline H3c: DISP $\rightarrow$ INSTIT & $0.373(10.937)^{* *}$ & $0.299^{* *}$ & $0.446^{* *}$ \\
\hline H4a: RECIP $\rightarrow$ TRUST & $0.229(4.053)^{* *}$ & 0.103 & $0.367^{* *}$ \\
\hline H4b: RECIP $\rightarrow$ INT & $0.129(2.401)^{*}$ & 0.088 & $0.198^{* *}$ \\
\hline H5a: BRAND $\rightarrow$ TRUST & $0.312(7.126)^{* *}$ & $0.406^{* *}$ & $0.227^{* *}$ \\
\hline H5b: BRAND $\rightarrow$ INT & $0.217(5.606)^{* *}$ & $0.335^{* *}$ & 0.108 \\
\hline H6a: CONTROL $\rightarrow$ TRUST & $-0.323(-4.638)^{* *}$ & $-0.260^{* *}$ & $-0.426^{* *}$ \\
\hline H6b: CONTROL $\rightarrow$ INT & $0.022(0.356)$ & 0.058 & -0.060 \\
\hline H7a: OFFLINE $\rightarrow$ TRUST & $0.130(1.380)$ & $0.208^{*}$ & -0.017 \\
\hline H7b: OFFLINE $\rightarrow$ INT & $-0.154(-1.866)$ & -0.145 & -0.327 \\
\hline H8a: ENDORS $\rightarrow$ TRUST & $0.214(2.134)^{*}$ & 0.130 & 0.381 \\
\hline H8b: ENDORS $\rightarrow$ INT & $-0.067(-0.658)$ & -0.137 & 0.150 \\
\hline & & & \\
\hline$R^{2} I N T$ & 0.727 & 0.771 & 0.658 \\
\hline$R^{2} T R U S T$ & 0.424 & 0.443 & 0.441 \\
\hline$R^{2}$ INSTIT $* .090$ & 0.199 \\
\hline$* \mathrm{p}<0.05 * \mathrm{p}<0.01$ & 0.139 & & \\
\hline
\end{tabular}

Table 1: Group Comparison of Beta-Coefficients and Total Variance Explained 